\title{
On a Necessary Requirement for Re-Uniform Numerical Methods to Solve Boundary Layer Equations for Flow along a Flat Plate
}

\author{
Grigorii I. Shishkin ${ }^{1}$, Paul A. Farrell ${ }^{2}$, Alan F. Hegarty ${ }^{3}$, John J. H. Miller ${ }^{4}$, \\ and Eugene O'Riordan ${ }^{5}$ \\ ${ }^{1}$ Institute of Mathematics and Mechanics, Russian Academy of Sciences, \\ Ural Branch, Ekaterinburg 620219, Russia \\ Grigorii@shishkin.ural.ru \\ 2 Department of Mathematics and Computer Science, Kent State University \\ Kent, Ohio 44242, USA \\ farrell@mcs. kent. edu \\ 3 Department of Mathematics and Statistics, University of Limerick \\ Limerick, Ireland \\ Alan.Hegarty@ul.ie \\ ${ }^{4}$ Department of Mathematics, University of Dublin, Trinity College \\ Dublin 2, Ireland \\ jmiller@tcd.ie \\ 5 School of Mathematical Sciences, Dublin City University \\ Dublin 9, Ireland \\ oriordae@ccmail.dcu.ie
}

\begin{abstract}
We consider grid approximations of a boundary value problem for the boundary layer equations modeling flow along a flat plate in a region excluding a neighbourhood of the leading edge. The problem is singularly perturbed with the perturbation parameter $\varepsilon=1 /$ Re multiplying the highest derivative. Here the parameter $\varepsilon$ takes any values from the half-interval $(0,1]$, and $R e$ is the Reynolds number. It would be of interest to construct an $R e$-uniform numerical method using the simplest grids, i.e., uniform rectangular grids, that could provide effective computational methods. To this end, we are free to use any technique even up to fitted operator methods, however, with fitting factors independent of the problem solution. We show that for the Prandtl problem, even in the case when its solution is self-similar, there does not exist a fitted operator method that converges Re-uniformly. Thus, combining a fitted operator and uniform meshes, we do not succeed in achieving $R e$-uniform convergence. Therefore, the use of the fitted mesh technique, based on meshes condensing in a parabolic boundary layer, is a necessity in constructing $R e$-uniform numerical methods for the above class of flow problems.
\end{abstract}

\section{Introduction}

The boundary layer equations for laminar flow are a suitable model for NavierStokes equations with large Reynolds numbers $R e$. Boundary value problems

L. Vulkov, J. Waśniewski, and P. Yalamov (Eds.): NAA 2000, LNCS 1988, pp. 723-731, 2001.

(C) Springer-Verlag Berlin Heidelberg 2001 
for these nonlinear equations are singularly perturbed, with the perturbation parameter $\varepsilon$ defined by $\varepsilon=R e^{-1}$. The presence of parabolic boundary layers, i.e., layers described by parabolic equations, is typical for such problems $[1,2]$.

The application of numerical methods, developed for regular boundary value problems (see, for example, $[3,11]$ ), even to linear singularly perturbed problems yields errors which essentially depend on the perturbation parameter $\varepsilon$. For small values of $\varepsilon$, the errors in such numerical methods may be comparable to, or even much larger than the exact solution. This behaviour of the approximate solutions creates the need to develop numerical methods with errors that are independent of the perturbation parameter $\varepsilon$, i.e. $\varepsilon$-uniform methods.

The presence of a nonlinearity in the differential equations makes it considerably more difficult to construct $\varepsilon$-uniform numerical methods. For example, even in the case of ordinary differential quasilinear equations, $\varepsilon$-uniform fitted operator methods (see, e.g., $[5,6]$ ) do not exist. It should be pointed out that even for linear singularly perturbed problems with parabolic boundary layers there are no $\varepsilon$-uniform fitted schemes (see, for example, [7-9]). Thus, the development of special $\varepsilon$-uniform numerical methods for resolving the Navier-Stokes and boundary layer equations has considerable scientific and practical interest.

In this paper, we consider grid approximations of a boundary value problem for boundary layer equations for a flat plate on a bounded domain outside a neighbourhood of its leading edge. The solution of the Prandtl problem is selfsimilar and exhibits a parabolic boundary layer in the considered domain. We study a wide class of discrete approximations consistent with the differential equations, i.e., the coefficients in the finite difference operators related to the differential coefficients do not depend on the problem solution. It is shown that the use of special meshes condensing in the boundary layer region is necessary. Also, no technique for the construction of the discrete equations leads to an $\varepsilon$-uniform method, unless it uses condensing grids.

\section{Problem Formulation}

Let us formulate a boundary value problem for Prandtl's boundary layer equations on a bounded domain. Consider a flat semi-infinite plate in the place of the semiaxis $P=\{(x, y): x \geq 0, y=0\}$. The problem is considered to be symmetric with respect to the plane $y=0$; we discuss the steady flow of an incompressible fluid on both sides of $P$, which is laminar and parallel to the plate (no separation occurs on the plate).

As is well known, singularities in such a problem arise for a large Re number. A typical singularity is the appearance of a parabolic boundary layer in a neighbourhood of the flat plate outside some neighbourhood of its leading edge. In a neighbourhood of the leading edge, another type of singularity is generated because the compatability conditions are violated at the leading edge. In order to concentrate on the boundary layer region under consideration, we skip a small neighbourhood of the leading edge. 
We consider the solution of the problem on the bounded set

$$
\bar{G}, \quad \text { where } G=\left\{(x, y): x \in\left(d_{1}, d_{2}\right], y \in\left(0, d_{0}\right)\right\}, \quad d_{1}>0
$$

with the boundary $S=\bar{G} \backslash G$. Let $G^{0}=\left\{(x, y): x \in\left[d_{1}, d_{2}\right], y \in\left(0, d_{0}\right]\right\}$; $\bar{G}^{0}=\bar{G}$; and let $S^{0}=\bar{G} \backslash G^{0}$ be the boundary of the set $G^{0}$. Assume $S=\cup S_{j}$, $j=0,1,2$, where $S_{0}=\left\{(x, y): x \in\left[d_{1}, d_{2}\right], y=0\right\}, S_{1}=\{(x, y): x=$ $\left.d_{1}, y \in\left(0, d_{0}\right]\right\}, S_{2}=\left\{(x, y): x \in\left(d_{1}, d_{2}\right], y=d_{0}\right\}, \bar{S}_{0}=S_{0}$. Thus, the boundary $S^{0}=S_{0}$ belongs to $P$. On the set $\bar{G}$, it is required to find a function $U(x, y)=(u(x, y), v(x, y))$ which is the solution of the following Prandtl problem:

$$
\begin{aligned}
L^{1}(U(x, y)) & \equiv \varepsilon \frac{\partial^{2}}{\partial y^{2}} u(x, y)-u(x, y) \frac{\partial}{\partial x} u(x, y)- \\
-v(x, y) \frac{\partial}{\partial y} u(x, y)=0, \quad(x, y) \in G, & (x, y) \in G^{0}, \\
L^{2} U(x, y) & \equiv \frac{\partial}{\partial x} u(x, y)+\frac{\partial}{\partial y} v(x, y)=0, \quad(x, y) \in S, \\
u(x, y) & =\varphi(x, y), \quad(x, y) \in S^{0} .
\end{aligned}
$$

Here $\varepsilon=R e^{-1}$; the parameter $\varepsilon$ takes arbitrary values in the half-interval $(0,1]$.

We now wish to define the functions $\varphi(x, y)$ and $\psi(x, y)$ more precisely.

In the quarter plane

$$
\bar{\Omega}, \text { where } \Omega=\{(x, y): x, y>0\}
$$

we consider the following Prandtl problem which has a self-similar solution [1]:

$$
\begin{aligned}
& L^{1}(U(x, y))=0, \quad(x, y) \in \Omega, \quad L^{2} U(x, y)=0, \quad(x, y) \in \bar{\Omega} \backslash P, \\
& u(x, y)=u_{\infty}, \quad x=0, \quad y \geq 0, \quad U(x, y)=(0,0), \quad(x, y) \in P .
\end{aligned}
$$

The solution of problem (4), (3) can be written in terms of some function $f(\eta)$ and its derivative

$$
u(x, y)=u_{\infty} f^{\prime}(\eta), \quad v(x, y)=\varepsilon^{1 / 2}\left(2^{-1} u_{\infty} x^{-1}\right)^{1 / 2}\left(\eta f^{\prime}(\eta)-f(\eta)\right)
$$

where $\eta=\varepsilon^{-1 / 2}\left(2^{-1} u_{\infty} x^{-1}\right)^{1 / 2} y$. The function $f(\eta)$ is the solution of the Blasius problem

$$
\begin{gathered}
L(f(\eta)) \equiv f^{\prime \prime \prime}(\eta)+f(\eta) f^{\prime \prime}(\eta)=0, \quad \eta \in(0, \infty), \\
f(0)=f^{\prime}(0)=0, \quad \lim _{\eta \rightarrow \infty} f^{\prime}(\eta)=1 .
\end{gathered}
$$

The functions $\varphi(x, y), \psi(x, y)$ are defined by ${ }^{1}$

$$
\varphi(x, y)=u_{(5)}(x, y), \quad(x, y) \in S ; \quad \psi(x, y)=v_{(5)}(x, y), \quad(x, y) \in S^{0}
$$

note that $\varphi(x, y)=0, \quad \psi(x, y)=0, \quad(x, y) \in S^{0}$.

\footnotetext{
${ }^{1}$ Here and below the notation $w_{(j . k)}$ indicates that $w$ is first defined in equation (j.k).
} 
In the case of problem (2), (7), (1), as $\varepsilon$ tends to zero, a parabolic boundary layer appears in a neighbourhood of the set $S^{0}$.

To solve problem (2), (7), (1) numerically, we wish to construct an $\varepsilon$-uniform finite difference scheme.

\section{Classical Difference Scheme for the Prandtl Problem}

For the boundary value problem $(2),(7),(1)$ we use a classical finite difference scheme. At first we introduce the rectangular grid on the set $\bar{G}$ :

$$
\bar{G}_{h}=\bar{\omega}_{1} \times \bar{\omega}_{2}
$$

where $\bar{\omega}_{1}$ and $\bar{\omega}_{2}$ are meshes on the segments $\left[d_{1}, d_{2}\right]$ and $\left[0, d_{0}\right]$, respectively; $\bar{\omega}_{1}=\left\{x^{i}: \quad i=0, \ldots, N_{1}, x^{0}=d_{1}, x^{N_{1}}=d_{2}\right\}, \bar{\omega}_{2}=\left\{y^{j}: \quad j=0, \ldots, N_{2}, y^{0}=\right.$ $\left.0, y^{N_{2}}=d_{0}\right\} ; N_{1}+1$ and $N_{2}+1$ are the number of nodes in the meshes $\bar{\omega}_{1}$ and $\bar{\omega}_{2}$. Define $h_{1}^{i}=x^{i+1}-x^{i}, x^{i}, x^{i+1} \in \bar{\omega}_{1}, h_{2}^{j}=y^{j+1}-y^{j}, y^{j}, y^{j+1} \in \bar{\omega}_{2}$, $h_{1}=\max _{i} h_{1}^{i}, h_{2}=\max _{j} h_{2}^{j}, h=\max \left[h_{1}, h_{2}\right]$. We assume that ${ }^{2} h \leq M N^{-1}$, where $N=\min \left[N_{1}, N_{2}\right]$.

We approximate the boundary value problem by the difference scheme

$$
\begin{aligned}
\Lambda^{1}\left(U^{h}(x, y)\right) & \equiv \varepsilon \delta_{\bar{y} \widehat{y}} u^{h}(x, y)-u^{h}(x, y) \delta_{\bar{x}} u^{h}(x, y)- \\
-v^{h}(x, y) \delta_{\bar{y}} u^{h}(x, y)=0, \quad(x, y) \in G_{h}, & \\
\Lambda_{1}^{2} U^{h}(x, y) & \equiv \delta_{\bar{x}} u^{h}(x, y)+\delta_{\bar{y}} v^{h}(x, y)=0, \quad(x, y) \in G_{h}^{0}, \quad x>d_{1}, \\
\Lambda_{2}^{2} U^{h}(x, y) & \equiv \delta_{x} u^{h}(x, y)+\delta_{\bar{y}} v^{h}(x, y)=0, \quad(x, y) \in S_{1 h} ; \\
u^{h}(x, y) & =\varphi(x, y), \quad(x, y) \in S_{h}, \\
v^{h}(x, y) & =\psi(x, y), \quad(x, y) \in S_{h}^{0} .
\end{aligned}
$$

Here $\delta_{\bar{y} \widehat{y}} z(x, y)$ and $\delta_{x} z(x, y), \ldots, \delta_{\bar{y}} z(x, y)$ are the second and first (forward and backward) difference derivatives (the bar denotes the backward difference), as follows: $\delta_{\bar{y} \widehat{y}} z(x, y)=2\left(h_{2}^{j-1}+h_{2}^{j}\right)^{-1}\left(\delta_{y} z(x, y)-\delta_{\bar{y}} z(x, y)\right), \quad \delta_{x} z(x, y)=$ $\left(h_{1}^{i}\right)^{-1}\left(z\left(x^{i+1}, y\right)-z(x, y)\right), \ldots, \delta_{\bar{y}} z(x, y)=\left(h_{2}^{j-1}\right)^{-1}\left(z(x, y)-z\left(x, y^{j-1}\right)\right)$, $(x, y)=\left(x^{i}, y^{j}\right)$.

The difference scheme (2), (1) approximates problem (2), (1) with the first order of accuracy for a fixed value of the parameter $\varepsilon$.

When the "coefficients" multiplying the differences $\delta_{\bar{x}}$ and $\delta_{\bar{y}}$ in the operator $\Lambda^{1}$ are known (let these be the functions $u_{0}^{h}(x, y)$ and $v_{0}^{h}(x, y)$ ), and if they satisfy the condition $u_{0}^{h}(x, y), v_{0}^{h}(x, y) \geq 0, \quad(x, y) \in \bar{G}_{h}$, we know that the operator $\Lambda^{1}$ is monotone [4]. In such a case we say that the discrete momentum equation (2a) is monotone.

It is known that even for linear singularly perturbed problems, if we consider the heat equation with $y$ and $x$ being the space and time variables, the errors of

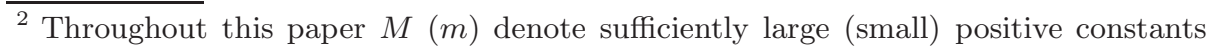
independent of $\varepsilon$ and the discretization parameters.
} 
the discrete solution depend on the perturbation parameter and become comparable with the exact solution itself when the value $\varepsilon^{1 / 2}$ has the same order of magnitude as the mesh step-size $h_{2}$ on uniform grids. Therefore, it is not surprising that for the solution of the difference scheme (2), (1) we have the following lower bounds

$$
\max _{\bar{G}_{h}}\left|u(x, y)-u^{h}(x, y)\right| \geq m, \quad \max _{\bar{G}_{h}}\left|v^{*}(x, y)-v^{* h}(x, y)\right| \geq m .
$$

Here $v^{*}(x, y)=\varepsilon^{-1 / 2} v(x, y), v^{* h}(x, y)=\varepsilon^{-1 / 2} v^{h}(x, y)$. We call the functions $u(x, y), u^{h}(x, y)$ and $v^{*}(x, y), v^{* h}(x, y)$ the normalized components of the solutions of problems (2), (7), (1) and (2), (1). Note that the functions $u(x, y), v^{*}(x, y)$ are $\varepsilon$-uniformly bounded on $\bar{G}$ and have order of unity, whereas the function $v(x, y)$ tends to zero when $\varepsilon \rightarrow 0$.

Thus, our aim is to try to find a numerical method which yields the discrete solutions $u^{0 h}(x, y), v^{0 * h}(x, y)$ satisfying the error estimates

$$
\begin{aligned}
& \left|u(x, y)-u^{0 h}(x, y)\right| \leq M\left[N_{1}^{-\nu_{1}}+N_{2}^{-\nu_{2}}\right], \\
& \left|v^{*}(x, y)-v^{0 * h}(x, y)\right| \leq M\left[N_{1}^{-\nu_{1}}+N_{2}^{-\nu_{2}}\right], \quad(x, y) \in \bar{G}_{h}^{0}
\end{aligned}
$$

where $\bar{G}_{h}^{0}$ is some grid on $\bar{G}$, and $u^{0 h}(x, y), v^{0 h}(x, y),(x, y) \in \bar{G}_{h}^{0}$ is the solution of some discrete problem on $\bar{G}_{h}^{0}, \nu_{1}$ and $\nu_{2}$ are any positive $\varepsilon$-independent numbers. Throughout this paper $M(m)$ denote sufficiently large (small) positive constants which do not depend on $\varepsilon$ and on the discretization parameters.

We say that the method is $\varepsilon$-uniform if the errors in the discrete normalized solutions are independent of the parameter $\varepsilon$. By a robust layer-resolving method we mean a numerical method that generates approximate normalized solutions that are globally defined, pointwise-accurate and parameter-uniformly convergent at each point of the domain, including the boundary layers. Thus, the errors of these normalized solutions in the $L_{\infty}$-norm are independent of $\varepsilon$, they depend only on $N_{1}$ and $N_{2}$ and tend to zero when $N_{1}, N_{2} \rightarrow \infty$.

Here we try to find a robust layer-resolving numerical method for the particular problem (2), (7), (1). It would be attractive to find a method using uniform grids. Obviously the method (2), (1) is too simple to be a robust layer-resolving method.

\section{On Fitted Operator Schemes for the Prandtl Problem}

In order to have freedom as much as possibly in the construction of an $\varepsilon$-uniform method, we use a fitted operator method. Such a method admits any technique to be used to construct discrete approximations to the solution of problem (2), (7), (1).

Before we proceed, we make a few remarks.

As was shown in $[8,10]$ (see also $[7,9,11]$ ) for a singularly perturbed parabolic equation with parabolic boundary layers, there exist no fitted operator schemes on uniform meshes that are $\varepsilon$-uniform. Note that the coefficients in the terms 
with first-order derivatives in time and second-order derivatives in the space variables do not vanish in the equations discussed in $[8,10]$. However, for the Prandtl problem the coefficient multiplying the first derivative with respect to the variable $x$, which plays the role of the time variable, vanishes on the boundary lying on the $x$-axis. Unlike the problems studied in $[8,10]$, where the boundary conditions do not obey any restriction, besides the requirement of sufficient smoothness, problem (2), (7), (1) is essentially simpler. Its solution depends only on the one parameter $u_{\infty}$. In [12] an $\varepsilon$-uniform fitted operator method was constructed for a linear parabolic equation with a discontinuous initial condition in the presence of a parabolic (transient) layer. Such fitted operator schemes have been successfully constructed because all of the singular components of the solution (their main parts) are defined, up to some multiplier, by just one function. Because of the simple (depending on $u_{\infty}$ only) representation of the solution for the Prandtl problem, it is not obvious that for this problem there are no $\varepsilon$-uniform fitted operator schemes. So it is of interest to establish whether such fitted operator schemes on uniform meshes do exist for the Prandtl problem.

We try to construct a fitted operator scheme starting from equation (2a) under the simplifying assumption that the function $v^{h}(x, y)$ is known, and also that $v^{h}(x, y)=v(x, y)$. Let us consider a fitted operator scheme of the form

$$
\begin{aligned}
& \Lambda^{1 *}\left(u^{h}(x, y)\right) \equiv \varepsilon \gamma_{(2)} \delta_{y} \bar{y} u^{h}(x, y)-u^{h}(x, y) \delta_{\bar{x}} u^{h}(x, y)- \\
&-\gamma_{(1)} v(x, y) \delta_{\bar{y}} u^{h}(x, y)=0, \quad(x, y) \in G_{h}, \\
& u^{h}(x, y)=\varphi(x, y), \quad(x, y) \in S_{h}
\end{aligned}
$$

where

$$
\bar{G}_{h} \text { is a uniform rectangular grid }
$$

with steps $h_{1}$ and $h_{2}$ in $x$ and $y$ respectively; the parameters

$$
\gamma_{(i)}=\gamma_{(i)}\left(x, y ; \varepsilon, h_{1}, h_{2}\right), \quad i=1,2
$$

are the fitting coefficients. The discrete equation (1a) is based on the classical monotone discrete momentum equation (2a). We emphasize that $\gamma_{(i)}$ are independent of the unknown solution that is defined by the parameter $u_{\infty}$. Note that equation $(2 \mathrm{a})$ is a particular grid equation from the class of discrete approximations (1a), (1c), namely, when $\gamma_{(i)}=1, i=1,2$.

Relying on a priori estimates for the solution of problem (2), (7), (1) and its derivatives, we establish, in a similar manner as in $[8,13]$, that there is no $\varepsilon$-uniform fitted operator scheme of the form (1), (2).

Theorem 1. In the class of finite difference schemes (1), (2) there exists no scheme whose solutions converges $\varepsilon$-uniformly, as $N \rightarrow \infty$, to the solution of the boundary value problem (2), (7), (1).

Remark 1. We conclude that to construct an $\varepsilon$-uniform scheme for the Prandtl problem (2), (1), provided that the coefficients $\gamma_{(i)}$ are independent of the problem solution, it is necessary to use meshes condensing in the neighbourhood of the parabolic boundary layer. No matter, whether finite elements or finite differences are used. 


\section{Condensing Mesh Technique}

Here we briefly describe the approach to the construction of an $\varepsilon$-uniform method with piecewise uniform condensing meshes that originated in [8].

We introduce a piecewise uniform mesh, which is refined in a neighbourhood of the boundary layer, i.e. of the set $S^{0}$. On the set $\bar{G}$, we consider the grid

$$
\bar{G}_{h}^{*}=\bar{\omega}_{1} \times \bar{\omega}_{2}^{*}
$$

where $\bar{\omega}_{1}$ is a uniform mesh on $\left[d_{1}, d_{2}\right], \bar{\omega}_{2}^{*}=\bar{\omega}_{2}^{*}(\sigma)$ is a special piecewise uniform mesh depending on the parameter $\sigma$ and the value $N_{2}$. The mesh $\bar{\omega}_{2}^{*}$ is constructed as follows. We divide the segment $\left[0, d_{0}\right]$ in two parts $[0, \sigma]$ and $\left[\sigma, d_{0}\right]$. The step-size of the mesh $\bar{\omega}_{2}^{*}$ is constant on the segments $[0, \sigma]$ and $\left[\sigma, d_{0}\right]$, given by $h_{2}^{(1)}=2 \sigma N_{2}^{-1}$ and $h_{2}^{(2)}=2\left(d_{0}-\sigma\right) N_{2}^{-1}$, respectively. The value of $\sigma$ is defined by

$$
\sigma=\min \left[2^{-1} d_{0}, m \varepsilon^{1 / 2} \ln N_{2}\right]
$$

where $m$ is an arbitrary positive number.

In the case of the boundary value problem $(2),(7),(1)$, it is required to study whether the solutions of the difference scheme (2), (1) converge to the exact solution.

We mention certain difficulties that arise in the analysis of the convergence.

Note that the difference scheme (2), (1), as well as the boundary value problem (2), (1), is nonlinear. To find an approximate solution of this scheme, we must construct an appropriate iterative numerical method. It is of interest to investigate the influence of the parameter $\varepsilon$ upon the number of iterations required for the convergence of this iterative process.

In the case of $\varepsilon$-uniform difference schemes for linear singular perturbation problems, methods are well developed to determine theoretically and numerically the parameters in the error bounds (orders of convergence and error constants) for fixed values of $\varepsilon$ and also $\varepsilon$-uniformly (see and compare, for example, [14,15]). In this technique, $\varepsilon$-uniform convergence is ascertained from theoretical investigations. Formally these methods are inapplicable to problem (2), (7), (1) because $\varepsilon$-uniform convergence of scheme (2), (1) was not justified by theory. Nevertheless, the results of such investigations of the error bounds seem to be interesting for practical use.

In [16] we give an experimental technique to study if this method is $\varepsilon$-uniform, and if so, to find the $\varepsilon$-uniform order of convergence. Some relevant ideas are discussed in [13]. This technique is used in [16] to analyze the scheme (2), (1). It was shown that this scheme gives discrete solutions that allow us to approximate the normalized component and its first derivatives in $x, y$ for problem (2), (7), (1). The order of $\varepsilon$-uniform convergence is close to one.

\section{Conclusion}

We have discussed the problems that arise when the direct method is designed to solve the Prandtl problem for flow along a flat plate. We have shown that, 
using a uniform mesh, it is impossible to construct a $R e$-uniform method if the coefficients of the fitted operator are independent of the problem solution. For such operators, in particular, for classical finite difference operators, the use of the grids condensing in the boundary layer region is necessary for the method to be $R e$-uniform.

\section{Acknowledgements}

This research was supported in part by the Russian Foundation for Basic Research under grant No. 98-01-00362, by the National Science Foundation grant DMS-9627244 and by the Enterprise Ireland grant SC-98-612.

\section{References}

1. Schlichting, H.: Boundary-Layer Theory. McGraw-Hill, New York (1979) 724, 725

2. Oleinik, O. A., Samohin, V. N: Mathemathical Methods in the Theory of Boundary Layer. CRC Press, Boca Raton (2000) 724

3. Marchuk, G. I.: Methods of Numerical Mathematics. Springer, New York (1982) 724

4. Samarskii, A. A.: Theory of Difference Schemes (in Russian). Nauka, Moscow (1989); German transl. Leipzig Akademische Verlag, Geest \& Portig (1984) 726

5. Shishkin, G. I.: Difference approximation of a singularly perturbed boundary value problem for quasilinear elliptic equations degenerating into a first-order equation. USSR Comput. Maths. Math. Phys. 32 (1992) 467-480 724

6. Farrell, P. A., Miller, J. J. H., O'Riordan, E., Shishkin, G. I.: On the non-existence of $\varepsilon$-uniform finite difference methods on uniform meshes for semilinear two-point boundary value problems. Math. Comp. 67 (222) (1998) 603-617 724

7. Shishkin, G. I.: Approximation of solutions of singularly perturbed boundary value problems with a parabolic boundary layer. USSR Comput. Maths. Math. Phys. 29 (1989) 1-10 727

8. Shishkin, G. I.: Grid Approximations of Singularly Perturbed Elliptic and Parabolic Equations (in Russian). Ural Branch of Russian Acad. Sci., Ekaterinburg (1992) $727,728,729$

9. Miller, J. J. H., O'Riordan, E., Shishkin, G. I.: Fitted Numerical Methods for Singular Perturbation Problems. Error Estimates in the Maximum Norm for Linear Problems in One and Two Dimensions. World Scientific, Singapore (1996) 727

10. Shishkin, G. I.: A difference scheme for a singularly perturbed equation of parabolic type with a discontinuous initial condition. Soviet Math. Dokl. 37 (1988) 792-796 727,728

11. Roos, H.-G., Stynes, M., Tobiska, L.: Numerical Methods for Singularly Perturbed Differential Equations. Convection-Diffusion and Flow Problems. Springer-Verlag, Berlin (1996) 724, 727

12. Hemker, P. W., Shishkin, G. I.: Discrete approximation of singularly perturbed parabolic PDEs with a discontinuous initial condition. Comp. Fluid Dynamics J. 2 (1994) 375-392 728 
13. Farrell, P. A., Hegarty, A. F., Miller, J. J. H., O’Riordan, E., Shishkin, G. I.: Numerical analysis of $R e$-uniform convergence for boundary layer equations for a flat plate. Computing (to appear). 728, 729

14. Shishkin, G. I.: Grid approximation of singularly perturbed parabolic equations degenerating on the boundary. USSR Comput. Maths. Math. Phys. 31 (1991) 53-63 729

15. Hegarty, A. F., Miller, J. J. H., O’Riordan, E., Shishkin, G. I.: Numerical solution of elliptic convection-diffusion problems on fitted meshes. CWI Quarterly 10 (1997) 239-251 729

16. Farrell, P. A., Hegarty, A. F., Miller, J. J. H., O’Riordan, E., Shishkin, G. I.: Robust Computational Techniques for Boundary Layers. CRC Press, Boca Raton (2000). 729 